\title{
Applying Genetic Algorithm for Capacitated Vehicle Routing Problem
}

\author{
Chunyu REN ${ }^{1, a}$ \\ ${ }^{1}$ School of Information science and technology, Heilongjiang University, Harbin, China \\ arency2004@163.com
}

Keywords: CVRP; natural coding; retain the best selection; 2-exchange mutation operator; improved route crossover operation

\begin{abstract}
The present study is focused on the Capacitated Vehicle Routing Problem (CVRP). Genetic algorithm is used to get the optimization solution. First of all, use natural number coding so as to simplify the problem; apply insertion method so as to improve the feasibility; retain the best selection so as to guard the diversity of group; adopt 2- exchange mutation operator to strengthen the partial searching ability of chromosome. Secondly, the improved route crossover operation can avoid destroying good gene parts. Finally, the good performance of improved algorithm can be proved by experiment calculation and concrete examples.
\end{abstract}

\section{Introduction}

Capacitated Vehicle Routing Problem is one of important researches in the fields of logistics distribution. Optimizing this problem can increase economic efficiency and satisfy the diversified and individual needs of customers.

When getting the solution of CVRP with big scale and multi-restricted condition, intelligent optimization method can get the satisfied and flexible solution in the limited time. BRAMEL presented a general framework for the capacitated vehicle routing problem [1]. Baker conducted a research on VRP problem with indefinite number of vehicles and used improved genetic algorithm to solve the problem [2]. Jung S modified GA's natural crossover operator, applied 2D image and implemented crossover operation to cut chromosome [3]. Bouthillier obtained initial solution through 2-opt and 3-opt, and then utilized multiple search algorithm featured by parallel cooperation to design crossover operator and mutation operator [4]. William applied C-W savings algorithm and the nearest neighbor search algorithm to construct initial solution, and then used GA to solve the VRP problem featured by many depots [5]. Geonwook applied hybrid GA to solve the VRP problem featured by many types of vehicles [6]. Simulated annealing algorithm has the characteristics of quick convergent speed and searching as whole through combination with other algorithms. Osman solved CVRP through simulated annealing and tabu search algorithms [7]. Chen designed the improved ant colony algorithm to solve CVRP [8].

Considering the complexity of CVRP, this article established the model and designed genetic algorithm to solve it. Computational experiments show that this algorithm is an efficient method for CVRP through a set of standard test problems.

\section{Model}

$$
Z=\operatorname{Min}\left\{\operatorname{Max} \sum_{i \in S} \sum_{j \in S} \sum_{k \in V} X_{i j k} d_{i j}\right\}
$$

Restraint condition,

$$
\begin{aligned}
& \sum_{k \in V} Y_{i k}=1, \quad i \in H . \\
& \sum_{i \in H} \sum_{j \in S} q_{i} X_{i j k} \leq W_{k}, \quad k \in V . \\
& \sum_{i \in S} X_{i j k}=Y_{i k}, \quad j \in S, \quad(2)
\end{aligned}
$$




$$
\begin{aligned}
& \sum_{j \in S} X_{i j k}=Y_{i k}, \quad i \in S, \quad k \in V . \\
& \sum_{i \in S} \sum_{j \in S} X_{i j k} \leq|m|-1, \quad \forall m \subseteq\{2,3 \ldots, n\}, \quad k \in V .
\end{aligned}
$$

In the formula: $G\left\{g_{r} \mid r=1, \ldots, R\right\}$ is a series of aggregations of distribution centre in the place $R$ (this essay only has one); $H\{h \mid i=R+1, . . R+N\}$ is a series of clients' aggregations in the place $N ; S\{G\} \cup\{H\}$ is the combination of all distribution centers and clients. $V\left\{v_{k} \mid k=1, . . K\right\}$ is travel vehicle $k$ 's aggregation; $q_{i}$ is the demand amount of client $i(i \in H) ; W_{k}$ is travel vehicle $k$ 's loading capacity; $d_{i j}$ is the linear distance from client $i$ to client $j ; D_{k}$ is the travel vehicle $k$ 's maximum travel mileage.

\section{Application in CVRP of Genetic Algorithm}

Genetic Coding. Adopt natural number coding and utilize one structure body to express the information of each supply point.

Initial Solution Forming. Supposed $h_{k}$ is the total number of all customer points for vehicle $k$. Muster $R_{k}=\left\{y_{i k} \mid 0 \leq i \leq h_{k}\right\}$ is the customer point of vehicle k. $Y_{i k}$ is the transportation tool of vehicle at i point. $Y_{0 k}$ is the distribution centre of starting point for vehicle $\mathrm{k}$. The concrete steps are as followings.

Step 1: Supposed the initial residual capacity of transportation vehicle as $w_{k}^{1}=w_{k}, k=0, h_{k}=0, R_{k}=\Phi$.

Step 2: The demand amount of $\mathrm{i}$ gene in a chromosome is $q_{i}$ and $k=1$.

Step 3: If $q_{i} \leq w_{k}^{1}, w_{k}^{1}=\operatorname{Min}\left\{\left(w_{k}^{1}-q_{i}\right), w_{k}\right\}$. Otherwise, it shifts to step 6 .

Step 4: If $w_{k}^{1}-q_{i} \leq w_{k}$ and $D_{i-1}+D_{i} \leq D_{k}, R_{k}=R_{k} \cup\{i\}$ and $h_{k}=h_{k}+1$. Otherwise, it shifts to step 6 .

Step 5: If $k>K, k=K$. Otherwise, $k=k$.

Step 6: $k=k+1$, shift to step 3 .

Step $7: i=i+1$, shift to step 2 .

Step 8: Repeat from step 2 to step 7. K memorizes the total amount of all vehicles. $R_{k}$ memorizes a group of feasible path.

Select Operator. The concrete steps are as followings.

Step1: Calculate the fitness value $f_{i}$ of each individual for community. Suppose that the highest fitness value of actual group is $f_{\text {best }}$.

Step2: Calculate the total fitness value $\sum f_{i}$.

Step3: Calculate the corresponding fitness value $p_{i}=\frac{f_{i}}{\sum f_{i}}$ of each individual in community.

Step4: Randomly create $\alpha$ in $[0,1]$. If $p_{1}+\ldots+p_{i-1}<\alpha<p_{1}+\ldots+p_{i-1}+p_{i}$, select individual i enter into the next generation community.

Crossover Operator. The improved route crossover operation is as followings.

Step1: Randomly select two chromosomes in father generation. According to conventional mode, get two groups of feasible vehicle routes after having feasible research on routes.

Step2: Generate mode $\mathrm{H}$ to every group of vehicle route, which mode is a bunch of randomly generating binary system number, its size is the amount of every groups of vehicles in length, that is, every binary system location is corresponding to one vehicle route.

Step3: In one father generation $P_{i}(i=1,2)$, to " 1 " route in mode $\mathrm{H}$, directly copy it to its filial generation $\mathrm{S}$. To " 0 " route in mode $\mathrm{H}$, form into one new order according to its successive sequence in another father generation $P_{j}(j=1,2, j \neq i)$.

Step4: To every requirement dot in 1 , successively insert into the place of first satisfying restriction condition in their respective filial generation in term of their successive order in sequence. 
Step5: If one requirement dot cannot insert into any existing routes, it will generate one new route which requirement dot will be inserted into in order to guarantee the principle of all requirement dot are satisfied.

Step6: Repeat them in turn until all requirement dots in generating sequence are inserted into one route. After finishing this operation, generate two new generations $s_{1}, s_{2}$.

Mutation Operator. The mutation strategy of this study is adopted 2- commutation mutation strategy, namely, randomly selecting mutated individual chromosome according to some mutation probability and two gene locations in this chromosome, exchanging gene in two places and form into new gene clusters. If it is continuously appeared with zero code in gene clusters, exchange zero code and non-zero code in random place.

\section{Experimental Calculation and Analysis}

To test hybrid genetic algorithm performance, its large-scale vehicle routing problem can give better results. In this paper, Christofides and Elion's E-n51-k5 VRP test problems is to verify. The test question is: in a distribution system, there is a logistics center, 50 customer needs point of the task, vehicle capacity of 160 , vehicle distribution center is 5 . Other data can be found in other literature [1]. It is required that rationally arrange distribution vehicle so as to the shortest delivery mileage.

Solution of Genetic Algorithm. After many trails, adopt following parameters, group scale $N N=50$, maximum iterations are $t_{\max }=600$. Crossover operator is $p_{c}=0.85$. Randomly get the solutions for 10 times.

Here, the total distance of best solution is 524.032 and concrete running path can be seen in table 1 and figure 1.

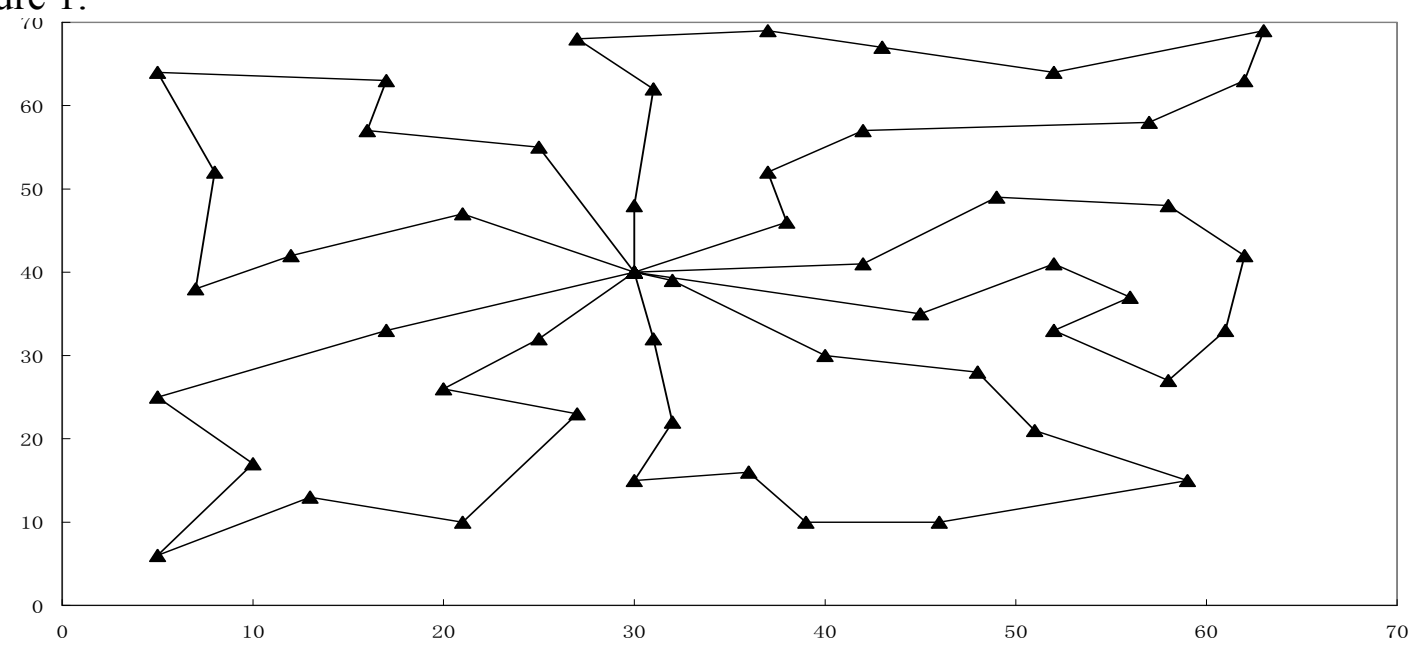

Fig. 1 Optimal routes on solving CVRP by GA

Table 1 Optimal result by GA

\begin{tabular}{|c|c|c|}
\hline No. & Running route & Distance \\
\hline 1 & $0-38-16-50-9-30-34-21-29-2-11-0$ & 99.532 \\
\hline 2 & $0-6-14-25-24-43-7-23-48-0$ & 97.661 \\
\hline 3 & $0-47-4-17-42-19-40-41-13-18-0$ & 109.056 \\
\hline 4 & $0-12-37-44-15-45-33-39-10-49-5-46-0$ & 99.251 \\
\hline 5 & $0-27-8-26-31-28-3-36-35-20-22-1-32-0$ & 118.532 \\
\hline \multicolumn{2}{|c|}{524.032} \\
\hline
\end{tabular}

Comparison with other algorithms. Compared the optimal scheme of reference $[1,2,7,8]$, experiments proved that this algorithm can achieve not only better calculating results, but also better calculation efficiency and quicker convergence rate. The concrete scheme can be shown in Table 2 .

Table2 Algorithm performance comparison

Algorithm

Optimal results 


\begin{tabular}{|c|c|}
\hline Savings Algorithm & 585 \\
\hline Tabu Search Algorithm & 536 \\
\hline Simulate Annealing Algorithm & 528 \\
\hline Ant Colony Algorithm & 524.61 \\
\hline This Algorithm & 524.032 \\
\hline
\end{tabular}

\section{Conclusions}

In all, this genetic algorithm proposed has much quicker convergence speed, stronger overcoming getting into partial optimal ability. Therefore, it is more practical significance and value so as to reduce operating cost and improve economic benefit.

\section{Acknowledgment}

This research was financially supported by the Ministry of Education of Humanities and Social Science Research Project of China (Grant NO. 12YJC630160).

\section{References}

[1] BRAMEL J, SIMCHI-LEVID. A location based heuristic for general routing problem. Operation Research, Vol. 43(1995), P. 649- 660

[2] BAKER B M. AYECHEW M A. A genetic algorithm for the vehicle routing problem. Computers \& Operations Research, Vol. 30(2003), P.787-800

[3] Jung. S, B. R. Moon. A hybrid genetic algorithm for the vehicle routing problem with time windows. Proceedings of the Genetic and Evolutionary Computation Conference, (2002), p.1309-1316

[4] Bouthillier, G Crainic. A cooperative parallel metaheuristic for vehicle routing with time windows. Computer \& Operation Research, Vol. 32(2005), p.1685-1708

[5] William Ho, George T S H, Ping Jib, Henry C W Lau. A hybrid genetic algorithm for the multi-depot vehicle routing problem. Engineering Applications of Artificial Intelligence, Vol. 21(2008), p.548-557

[6] Geonwook Jeon, Herman R Leep, Jae Young Shim. A vehicle routing problem solved by using a hybrid genetic algorithm. Computers \& Industrial Engineering, Vol. 53(2007), p. 680-692

[7] OSMAN I H. Metastrategy simulated annealing and tabu search algorithms for the vehicle routing problem. Annals of Operations Research, Vol. 41(1993), P. 421-451

[8] Chen $\mathrm{Ch} \mathrm{H}$, Ting Ch J. An improved ant colony system algorithm for the vehicle routing problem. Journal of the Chinese Institute of Industrial Engineer, Vol. 23(2006), P. 115-126 